\title{
Nuevos biomarcadores usados en el diagnóstico del daño renal en condiciones de estrés oxidativo
}

New biomarkers used in the diagnosis of kidney damage under conditions of oxidative stress.

\author{
Azucena E. Jiménez-Corona ${ }^{a}$, Gilberto Hernández-Hernández ${ }^{b}$, Luis R. Martínez-Santos ${ }^{b}$
}

\begin{abstract}
:
This study shows the importance of candidate biomarkers to predictors of kidney disease, through the results and evidence obtained in recent years, as well as the direct relationship with oxidative stress. Chronic Renal Disease (CKD), which is the fourth most prevalent worldwide and is defined as the progressive loss of kidney functionality and a sudden decrease in glomerular filtration $<60 \mathrm{ml} / \mathrm{min} / 1.73 \mathrm{~m} 2$, for 3 or more months, accompanied by renal abnormalities, structural, functional or both, with accumulation of nitrogen products and an alteration in hydroelectrolitic homeostasis with the passage of months and even years.
\end{abstract}

Keywords:

Chronic Renal Disease, Biomarkers, Oxidative Stress, Obesity, Glomerular filtration.

\section{Resumen:}

En este estudio se muestra la importancia de biomarcadores candidatos a predictores de enfermedad renal, mediante los resultados y evidencias obtenidas en los últimos años, así como también, la relación directa con el estrés oxidativo. La Enfermedad Renal Crónica (ERC), representa el cuarto lugar en prevalencia a nivel mundial y se define como la pérdida progresiva de la funcionalidad del riñón y una disminución brusca del filtrado glomerular $<60 \mathrm{ml} / \mathrm{min} / 1.73 \mathrm{~m} 2$, durante 3 o más meses, acompañada por anormalidades renales, de tipo estructural, funcional o ambas, con acumulo de productos nitrogenados y una alteración en la homeostasis hidroelectrolítica con el paso de los meses e incluso años.

Palabras Clave:

Enfermedad Renal Crónica, Biomarcadores, Estrés Oxidativo, Obesidad, Filtración Glomerular.

\section{Introducción}

Actualmente a nivel mundial, es bien conocido que el sedentarismo en la población combinado con dietas hipercalóricas conlleva al sobrepeso y a la obesidad, convirtiéndose así en factores importantes de riesgo para desencadenar el síndrome metabólico, y padecimientos múltiples incluyendo el progreso de enfermedades crónico degenerativas, como es el caso del daño cardiovascular y la enfermedad renal crónica (ERC). ${ }^{1}$ Este mecanismo es debido a que, en la población con obesidad se produce un mecanismo de hiperfiltración, probablemente compensatorio con el fin de satisfacer la alta demanda metabólica asociada al incremento de peso corporal, lo que ocasiona el aumento de la presión intraglomerular y como consecuencia el riesgo de desarrollar ERC. ${ }^{2}$

En base a lo anterior, la ERC se considera un problema de salud pública creciente, ya que, si ésta no es tratada de manera inmediata puede dar lugar a complicaciones graves como: enfermedades cardiovasculares, cerebrovasculares, trastornos hematológicos, minerales y óseos. ${ }^{3}$ Además del aumento de la mortalidad a causa de estas complicaciones. Asimismo la $\mathrm{ERC}$, se define como la pérdida

\footnotetext{
a Autor de correspondencia, Universidad Autónoma del Estado de Hidalgo, Escuela Superior de Huejutla, https://orcid.org/0000-0002-8987506X, Email: azucena_jimenez@uaeh.edu.mx

b Universidad Autónoma del Estado de Hidalgo, Escuela Superior de Huejutla, Email: he317379@uaeh.edu.mx

b Universidad Autónoma del Estado de Hidalgo, Escuela Superior de Huejutla, Email: ma317390@uaeh.edu.mx
} 
progresiva de la funcionalidad del riñón y una disminución brusca del filtrado glomerular $<60 \mathrm{ml} / \mathrm{min} / 1.73 \mathrm{~m}^{2}$ durante 3 o más meses, acompañada por anormalidades renales de tipo estructural, funcional o ambas, con acumulo de productos nitrogenados y una alteración en la homeostasis hidroelectrolítica a corto y mediano plazo. ${ }^{4,5}$

La ERC, evoluciona de forma asintomática sin detectarse hasta situaciones muy avanzadas, por lo que en la mayoría de los casos es una enfermedad difícilmente prevenible. Debido a esto, es de gran relevancia mejorar la detección temprana e influir en la progresión de la enfermedad renal, ya que así, se disminuirían las complicaciones cardiovasculares asociadas a la enfermedad renal y mejoraría el pronóstico, a corto y largo plazo. ${ }^{4}$ Por lo tanto, al ser la ERC el cuarto padecimiento con prevalencia a nivel mundial es necesario mejorar los métodos de diagnóstico tempranos dirigidos hacia esta patología. ${ }^{6}$

Por otro lado, una variable importante que nos permite determinar el daño renal es, la velocidad de filtración glomerular específica (VFGe), que se calcula empleando fórmulas que involucran la concentración de la creatinina plasmática y ciertas variables demográficas como: edad, sexo y raza. Sin embargo, actualmente los últimos hallazgos reportados, están relacionados con nuevos biomarcadores mayormente sensibles y específicos usados en la determinación oportuna del daño renal. ${ }^{7}$

\section{Antecedentes}

A nivel mundial un gran porcentaje de la población padecen ERC, debido al mal funcionamiento de los riñones derivado de un cuadro fisiopatológico previo a la disfunción. Hoy día, existen tratamientos sencillos y de bajo costo que pueden reducir el progreso de la enfermedad previniendo el riesgo de ataques cardiacos y accidentes cerebrovasculares.

Es importante destacar, que en etapas iniciales la enfermedad no presenta síntomas y puede ser tratada, sin embargo, en etapas avanzadas los pacientes pueden necesitar de terapia sustitutiva $y$ en un grado mayor un trasplante renal. ${ }^{4}$ Bjornstand y colaboradores en el 2018, determinaron la medición de algunos biomarcadores mediante muestras de plasma para determinar la lesión renal y así aumentar la predicción del padecimiento de la enfermedad renal en adultos con Diabetes tipo 1, sus resultados abarcaron los siguientes biomarcadores: Molécula de lesión renal-1(KIM 1), calbindina, osteoactivina, trébol factor 3 (TFF3), factor de crecimiento endotelial vascular (VEGF), microglobulina, cistatina C, Lipocalina asociada a gelatinasa de neutrófilos (NGAL) y osteopontina (OPN). ${ }^{8}$

De acuerdo a estos hallazgos, recientemente numerosas moléculas proteicas han sido identificadas como candidatos a biomarcadores de ERC. Particularmente la osteopontina, osteocalcina, y osteoprotegerina, las cuales, son proteínas presentes fisiológicamente en tejido óseo. Sin embargo, la aparición de estas proteínas en la excreción renal se encuentra aumentada en respuesta a la lesión renal, por lo tanto, son biomoléculas blanco en la predicción de esta patología. ${ }^{9}$

\section{Osteopontina}

La OPN es una proteína multifuncional presente en muchos tejidos y fluidos corporales como: plasma, orina, leche y bilis. ${ }^{10,11}$ Es producida por varios tipos de células, incluidas células inmunes como macrófagos activados y células $T$, células del cerebro y riñón, células de músculo liso vascular, mioblastos de la médula ósea, células dendríticas, hepatocitos, células neurales, así como; células involucradas en la morfogénesis ósea, tales como osteoblastos y osteoclastos ${ }^{12,13}$ La OPN regula diversas actividades biológicas, entre ellas la remodelación de la matriz y la calcificación tisular, la migración de monocitos/macrófagos en la quimiotaxis. ${ }^{14}$

Por otro lado, se sabe que los niveles de OPN se expresan en el Asa de Henle, en el túbulo contorneado proximal y distal, por lo que en estos segmentos se encuentran aumentados en 
modelos de nefrotoxicidad y por lo tanto, están relacionados en la lesión histológica. ${ }^{15}$

En el sistema renal, la OPN se sobreexpresa en pequeñas cantidades y se libera en la orina a través de las células epiteliales, por lo que, en una disfunción renal promueve la nucleación de cristales de fosfatos y oxalatos de calcio, dando como consecuencia la formación de cálculos renales. Estos precipitados (cálculos), obstruyen el flujo en las vías urinarias, por lo tanto, aumenta la presión en la cápsula de Bowman, disminuyendo la filtración glomerular, provocando así hidronefrosis (distensión y dilatación de la pelvis y los cálices renales). ${ }^{16}$

\section{Osteoprotegerina}

La Osteoprotegerina (OPG), es un receptor de citoquinas inflamatorias implicada en la remodelación ósea, asimismo es una glicoproteína soluble de la familia de receptores del factor de necrosis tumoral (TNF). ${ }^{16}$ La OPG se expresa en una variedad de tejidos, incluidos los endoteliales vasculares. ${ }^{9}$ En consecuencia, la OPG tiene doble función de protección: promueve la mineralización esquelética, pero previene la mineralización en el tejido vascular. ${ }^{17}$

Los niveles elevados de OPG circulantes en pacientes con enfermedades cardiovasculares y renales crónicas, se asocian de forma independiente con la mortalidad por causa del cáncer y la aterosclerosis. ${ }^{18,19}$

Evrard y colaboradores en el 2015, encontraron que la velocidad de pulso por onda no estaba relacionado con la OPG, sin embargo, se asoció positivamente con la edad y negativamente con el Índice de masa corporal (IMC). En este estudio postularon que, la edad y el IMC superaron los efectos de la OPG. Además determinaron que la OPG fosforilada es necesaria para lograr la inhibición de la Calcificación Vascular en comparación de la OPG no fosforilada. ${ }^{19}$ Hoy en día, todavía se discute acerca de la vía de expresión de la OPG en la lesión vascular, así como no está clara la relación de los altos niveles de OPG circulante con la regulación atenuante en el proceso de calcificación. ${ }^{9}$

\section{Osteocalcina}

La Osteocalcina $(O C)$ es una proteína de pequeño tamaño de la matriz extracelular de tipo no colágeno, donde es expresada en grandes cantidades por osteoblastos formadores de hueso. Tiene 45-50 residuos de aminoácidos que sufren modificaciones postraduccionales por vitamina $\mathrm{K}$ dependiente de $\mathrm{Y}$-carboxilación. Más allá de los efectos sobre su participación en la remodelación de los huesos, también regula la proliferación de células beta, la regulación de calcificación vascular, aterosclerosis, la expresión del gen de insulina, y la sensibilidad a la insulina, tales hallazgos fueron encontrados en estudios desarrollados en ratones y humanos. Asimismo, otras evidencias mostraron un papel potencial de la OC y su relación en el metabolismo de la glucosa y en la patogénesis de las alteraciones en diabetes mellitus tipo 2. ${ }^{20,21}$

En pacientes con la función renal alterada, los niveles plasmáticos de OC están notablemente elevados debido al aumento del recambio óseo y la eliminación renal disminuida. ${ }^{22}$ En otros estudios publicaron resultados que existe una relación positiva acerca de que la OC puede actuar como un predictor de enfermedades cardiovasculares y la mortalidad relacionada. En general, las anteriores evidencias han demostrado que las concentraciones bajas de OC en circulación están asociadas con un mayor riesgo de enfermedades cardiovasculares. ${ }^{23,24}$

De acuerdo a los hallazgos reportados por Lerchbaum $E$ et al 2013, observaron una relación inversa entre el riesgo de eventos cardiovasculares y los niveles plasmáticos de OC en pacientes con Hemodiálisis regular, lo que sugiere un papel protector vascular para la OC. ${ }^{25}$ Recientemente, se han proporcionado evidencias convincentes de que, la OC puede estar implicada en la Calcificación Vascular relacionada con el daño endotelial en pacientes con ERC. Dichos resultados demuestran que, las células endoteliales activadas en la uremia son capaces de producir macropartículas que inducen la expresión de OC en células progenitoras 
endoteliales y fibroblastos, así como en el desequilibrio, el proceso de reparación y daño endotelial. Por lo tanto la OC, puede contribuir a la Calcificación Vascular al aumentar el número de células progenitoras endoteliales positivas para OC. ${ }^{26}$

\section{Kim-1}

La molécula tipo1 de lesión renal (KIM-1) es expresada considerablemente en el túbulo proximal, particularmente en las zonas con mayor inflamación y daño del túbulo intersticial resultante de eventos de isquemia/reperfusión 0 nefrotoxicidad. En un estudio reciente, se demostró que los niveles de KIM-1 urinarios están relacionados con el riesgo de desarrollar insuficiencia renal crónica, independientemente del nivel de albuminuria. ${ }^{27}$

Notablemente, la alta expresión de Kim-1 se observa de forma característica en áreas de fibrosis e Inflamación. Por lo tanto, no es de extrañar que, en la nefropatía diabética los componentes patógenos incluyen fibrosis renal, expansión mesangial, hipertrofia glomerular y estrés oxidativo. ${ }^{28}$

Por otro lado, en el 2017 Cen y C y colaboradores observaron la expresión de KIM-1 como proteína transmembranal, encontrando que se expresa en bajas concentraciones en condiciones normales y se sobreexpresa en procesos de isquemia renal. 29

\section{Cistatina C}

La cistatina $C$ (Cys-C) es una pequeña molécula de $13 \mathrm{kDa}$ producida por todas las células nucleadas a un nivel constante, filtrada libremente por el glomérulo y casi completamente reabsorbida y degradada, pero no es secretada por las células tubulares proximales, por lo que habitualmente no se detecta en orina. Esta molécula ha sido identificada como un nuevo biomarcador para la detección temprana del daño renal precoz siendo está más sensible que la creatinina, ya que su expresión no se encuentra afectada por factores antropométricos como: la edad, sexo o la masa muscular. ${ }^{30,31}$

La concentración sérica de Cys- $C$ funciona como biomarcador lo cual permite estimar la tasa de filtración glomerular. Asimismo, se ha demostrado que la Cys-C, se encuentra alterada en ciertas afecciones inflamatorias $y$ trastornos del metabolismo. ${ }^{32}$

En condiciones normales más del $99 \%$ de cistatina (Cys-C) es filtrada por el glomérulo y reabsorbida en su totalidad por el túbulo proximal, en condiciones de ERC, la determinación de Cys$C$ en sangre, puede tener una mayor sensibilidad que la creatinina sérica para detectar cambios más sutiles y precoces en la función renal, por lo tanto, una elevación del $50 \%$ en el valor de Cys-C sérica puede predecir hasta 2 días antes del aumento en los niveles de creatinina sérica en la ERC. ${ }^{33}$

\section{NGAL}

Lipocalina asociada a gelatinasa de neutrófilos (NGAL) es una proteína de $20 \mathrm{kDa}$ expresada en los túbulos renales, esta glicoproteína se libera en la orina en la lesión renal aguda o crónica. ${ }^{34}$

En las últimas décadas, estudios en humanos mostraron evidencias de que cuando existe un aumento de 10 veces los valores de NGAL en plasma aumentarán 100 veces los valores en orina, en comparación de aquellos pacientes que no presentan ERC. Por lo tanto, las altas concentraciones de NGAL pueden reflejar una lesión renal de manera oportuna. ${ }^{35}$

En otro estudio, los pacientes pediátricos que recibieron un bypass cardiopulmonar en cursando con ERC, presentaron niveles aumentados de NGAL en plasma y en orina desde las primeras 2 horas post-cirugía, en comparación con la creatinina sérica que tardo entre 1 y 3 días en elevarse. ${ }^{36}$

\section{Estrés oxidativo}

El estrés oxidativo y la inflamación son características de la enfermedad renal crónica (ERC). La peroxidación de los lípidos de la membrana da como resultado la pérdida de 
fluidez, elasticidad de la membrana y funcionamiento celular desordenado. La oxidación de proteínas provoca la fragmentación de residuos de aminoácidos que conducen a enlaces cruzados, pérdida de configuración y funciones de proteínas. El daño oxidativo del ADN causa mutación en las bases del mismo por lo tanto, el aumento del estrés oxidativo en la enfermedad renal crónica se ha implicado aún más en la patogénesis de la calcificación vascular. Estudios realizados por Vera-Aviles $M$ et al 2018, demostraron que la histidina actúa como un protector contra el daño oxidativo, esto en pacientes con enfermedad renal crónica. Asimismo, se ha encontrado que L-histidina exhibe capacidades antioxidantes, como la eliminación de radicales libres y quelantes de iones metálicos divalentes, por lo tanto, su uso se encuentra benéfico para mejorar el estrés oxidativo en la ERC. ${ }^{37}$

El epitelio del túbulo renal es expuesto a daño por especies químicas, entre estas tenemos: a las lipoproteínas de baja densidad oxidadas (LDLOx), metales de transición, hemoglobina y mioglobina, o drogas potencialmente nefrotóxicas. Todo esto es debido, a que en la enfermedad renal crónica existe aparición de macromoléculas en el espacio urinario, ya que se pierde la selectividad del epitelio glomerular. Puesto que, la exposición de las células tubulares con las LDLOx podría resultar en el daño en el túbulointersticial, debido a la inducción de un ambiente pro-oxidante. El papel de las LDL nativas en la producción de estrés oxidativo ha sido claramente establecida, siendo la presencia de concentraciones aumentadas de LDL un factor de daño glomerular relevante. Este daño podría ser directo o indirecto, debido a que la oxidación de las LDL es inducida por la infiltración leucocitaria, resultando un incremento en el daño glomerular. Por lo tanto, el glomérulo es considerablemente más sensible al daño oxidativo que otros segmentos de la nefrona, estos hallazgos fueron reportados por Rodrigo Castillo et al 2003. ${ }^{38}$

En un estudio con 939 muestras de humanos, provenientes de biopsias renales, utilizaron anticuerpos monoclonales (mAbs) OL-10, 48 y
449, y un anticuerpo policlonal contra la apolipoproteína (apo) B. Mediante un análisis inmunocitoquímico, identificaron que el (6.6\%) de las muestras provenientes de 72 pacientes de los 939 con enfermedad renal, reportaron que, en los segmentos escleróticos 0 áreas mesangiales afectadas, debido a la internalización de las LDLOx, ya que estas se encontraron localizadas en las lesiones de glomeruloesclerosis y áreas mesangiales en biopsias renales humanas específicas para LDL-Ox y malondialdehído (MDA) en los glomérulos. ${ }^{39}$

Asimismo, la hiperlipidemia se asocia con una pérdida más rápida de la función renal, ya que los individuos con insuficiencia renal en su mayoría cursan con hiperlipidemia, aunque, no todos los pacientes con niveles elevados de lípidos tienen enfermedad renal, la hiperlipidemia puede exacerbar la insuficiencia renal preexistente por lesión directa en el basamento glomerular; Sandhu $S$ et al 2006, llevaron a cabo un metaanálisis con el objetivo de evaluar el efecto de las estatinas sobre el cambio en la función renal y la excreción de proteínas en la orina. Determinaron si el tratamiento con estatinas traía algún beneficio bajo esta condición de estrés, concluyendo que las estatinas parecen reducir la proteinuria moderadamente y produce una pequeña reducción en la tasa de pérdida de la función renal, especialmente en poblaciones con enfermedad cardiovascular. ${ }^{40}$

Gerard-Monnier y colaboradores en 1998 desarrollaron un nuevo ensayo colorimétrico de la peroxidación lipídica. Esto se realizó mediante reacciones cromogénicas, utilizando un medio a base de ácido metanosulfonico, MDA y 4hidroxialquenales. Dichos compuestos se pueden medir a una longitud de onda de $586 \mathrm{~nm}$, los cuales son altamente específicos $y$ comparables. ${ }^{41}$

En ciencias biológicas y médicas se ha demostrado que el malondialdehído es un biomarcador útil para la lipoperoxidación, por lo que existen diversos tipos de métodos analíticos para la medición del malondialdehído. Algunos métodos para la determinación de estrés oxidativo, se llevan a cabo por medio de análisis 
cromatográficos muy específicos y sensibles. Como por ejemplo: la detección de captura de electrones, la detección de fósforo nitrogenado y detección mediante fluorescencia. Esta técnica también se puede realizar utilizando diaminonaftaleno, hidralazina, ácido tiobarbitúrico, para la determinación de malondialdehído. ${ }^{42}$

\section{Proteínas con actividad antioxidante: PON-1, GST y NQO1}

La paraoxonasa de tipo 1 (PON-1) es una enzima que se encuentra ubicada principalmente en lipoproteínas de alta densidad, por lo que tiene un papel antioxidante que se refleja principalmente por la capacidad para hidrolizar los peróxidos de lípidos en partículas de LDL y HDL. Por lo tanto, la disminución de la actividad de la PON-1 se asocia con diferentes factores patológicos, los cuales desembocan a la ERC. ${ }^{43}$

Por otro lado, la glutatión transferasa del eritrocito (e-GST) es un miembro de una superfamilia de enzimas inducibles involucradas en la protección y desintoxicación celular. Una función prominente de estas enzimas es la conjugación de glutatión (GSH) a compuestos hidrófobos tóxicos proporcionados por un centro electrofílico. Esta reacción facilita su inactivación y eliminación renal de un gran número de toxinas. ${ }^{44}$

\section{Proteínas con actividad oxidante: NQO1 y Mieloperoxidasa}

Lind C et. Al. 1990 determinaron la actividad de la $\mathrm{NAD}(\mathrm{P}) \mathrm{H}$ deshidrogenasa quinona-reductasa-1 (NQO1), la cual midieron utilizando (4 $\mathrm{mM})$ de $\mathrm{NADPH}$, como donante de electrones y 2-metil-1, 4-naftoquinona (menadiona; 0,1 $\mathrm{mM}$ ) como aceptor de electrones. El ensayo usaron Tris- $\mathrm{HCl}$ $50 \mathrm{mM}, \mathrm{pH} 7,5$ con Triton-100X 0,08\% con el fin de mantener la reoxidación continua; asimismo incorporaron a la reacción al citocromo-c, formado por menadiol (38.5 $\mu \mathrm{M})$. De esta manera, realizaron un ensayo selectivo agregando dicumarol (100 uM, disuelto en Tris- $\mathrm{HCl} 50 \mathrm{mM}$, pH 7.5 sin Triton-100X). La reacción se inició mediante la adición de la enzima, seguida $550 \mathrm{~nm}$.
La actividad de la enzima se calculó a través de la diferencia en la absorbancia entre la muestra sin y la muestra con inhibidor. La pendiente de cada muestra se ajustó por la concentración de proteína plasmática. ${ }^{45}$

La Mieloperoxidasa (MPO) oxida las lipoproteínas de baja densidad (LDL), facilita su acúmulo en los macrófagos y la formación de células espumosas, estimula las metaloproteínas que degradan la cápsula fibrosa, facilita la rotura de la placa, exhibe propiedades prooxidantes e inhibe al óxido nítrico y reduce sus acciones vasodilatadoras, antiagregantes y antiinflamatorias, a la vez que facilita la expresión de diversos factores protrombóticos y antifibrinolíticos. Por lo tanto, podría ser un marcador que relacione el estrés oxidativo, la inflamación, la disfunción endotelial y el riesgo trombogénico, a la vez que confirma la importancia de los neutrófilos en la fisiopatología de la aterosclerosis. ${ }^{46}$

La actividad excesiva de la MPO puede inducir daño a tejidos por la producción de oxidantes $y$, consecuentemente, formar especies reactivas de lípidos y proteínas. En estudios recientes se ha demostrado que la exposición de lipoproteínas de baja densidad (LDL) a leucocitos activados produce, a través de la MPO, especies nitrogenadas y halogenadas que facilitan procesos de peroxidación lipídica, nitración proteica y conversión a formas proaterogénicas de LDL en la pared vascular. ${ }^{47}$

\section{Conclusión}

La sobreproducción de Especies Reactivas de Oxígeno y Nitrógeno a nivel de la pared vascular genera disfunción endotelial que incrementa el riesgo y desarrollo de enfermedades cardiovasculares y renales. Por lo tanto es importante continuar con la búsqueda de nuevos marcadores pro-oxidantes y biomarcadores que tengan mayor especificidad en la predicción de la ERC, y/o detección en estadios tempranos. 


\section{Referencias}

[1] Hall ME, do Carmo JM, da Silva AA, Juncos LA, Wang Z, Hall JE. Obesity, hypertension, and chronic kidney disease. Int $\mathrm{J}$ Nephrol Renovasc Dis 2014; 7:75-88.

[2] Kovesdy C, Susan F y Zoccali C, Obesity and kidney disease: Hidden consequences of the epidemic. J Ren Nutr 201727 (2): 75-77.

[3] Jha V, Garcia-Garcia G, Iseki K, Li Z, Naicker S, Platther B, Saran R, Wang AY, Yang CW. Chronic Kidney Disease: Global dimensión and perspectives. The Lancet 2013; 382(9888): 260-72.

[4]. Monedero P, Garcia Fernandez N, Perez Valdivieso J.R, Vives M, Lavilla J. Acute kidney injury. Rev Esp Anestesiol Reanim 2011; 58 : 365-374.

[5]. Martínez A, Górriz J, Bover J, Segura-de la Morena J, Cebollada J, Escalada J, Esmatjes E, Fácila L, Gamarra J, Gràcia S, Hernánd J-, José L. Caro L, Mazón P, Montañés R, Morales F, Muñoz M, Velasco P, Santiago A, Sánchez M, Suárez C, Tranche S. Consensus document for detection and management of chronic kidney disease. Nefrología 2014; 34 (2):243-62.

[6]. Washington, DC, 2014 (OPS/OMS). Recuperado de: https://www.paho.org/hq/index.php?option=com_content\&view=articl e\&id=9379:2014-kidney-disease-rising-among-seniors-diabeteshypertension\&Itemid $=1926 \&$ lang $=e s$

[7]. Orozco R. Recognition and prevention of chronic kidney disease (CKD). Revista médica clínica los condes 2010; 21:779-789.

[8]. Bjornstad P, Pyle L, Cherney D.Z.I, Johnson RJ, Sippl R, Wong R, Rewers M, Snell-bergeon JK. Plasma biomarkrs improve prediction of diabetic kidney disease in adults with type 1 diabetes over a 12-year follow-up: CACTI study. Nephrol Dial Transplant 2018; 33: 1189-1196.

[9]. Csiky B, Sági B, Peti A, Lakatos O, Prémusz V, Sulyok E. The Impact of Osteocalcin, Osteoprotegerin and Osteopontin on Arterial Stiffness in Chronic Renal Failure Patients on Hemodialysis. Kidney Blood Press Res 2017; 6:1312 1321 .

[10]. Chapman J, Miles PD, Ofrecio JM, Neels JG, Yu JG, Resnik JL Wilkes J, Talukdar S, Thapar D, Jhonson K, Sears DD. Osteopontin is required for the early onset of high fat diet-induced insulin resistance in mice. PLos ONE 2010; 5(11): 139-59.

[11]. Kiefer FW, Neschen S, Pfau B, Legerer P, Neuhofer A, Kahne M, Hrabe de Angelis M, Schlederer M, Mair M, Kener L, Plutzky J, Zeyda M, Stulnig TM. Osteopontin defiiency protects against obesity-induced hepatic steatosis and attenuates glucose production in mice. Diabetologia 2011; 54(8): 2132-2142.

[12]. Wen Y, Jeong S, Xia Q, Kong X. Role of osteopontin in liver diseases. International Journal of Biological Sciences 2016; 12(9): 1121-1128.

[13]. Kothari AN, Arff ML, Chang V, Blackwel RH, Syn WK, Zhang J, M Z, Kuo PC. Osteopontin a master regulator of epithelial mesenchymal transition. Journal of Clinical Medicine 2016; (5) 4-39.

[14]. Barreto R, Guevara M. Biomarkers of acute kidney injury: a « trending topic » in cirrhosis Gastroenterol Hepatol. 2013; 6:407-21

[15]. Icer MA, Gezmen-Karadag M. The multiple functions and mechanisms of osteopontin. Clin Biochem 2018; 59:17-24.

[16]. Schoppet M, Preissner KT, Hofbauer LC. RANK ligand and osteoprotegerin: paracrine regulators of bone metabolism and vascular function. Arterioscler Thromb Vasc Biol 2002; 22:549-553.

[17]. Bjerre M, Hilden J, Kastrup J, Skoog M, Hansen JF, Kolmos HJ, Jensen GB, Kjoller E, Winkel P, Flyvbjerg A, Gluud C, Claricor TG. Osteoprotegerin independently predicts mortality in patients with stable coronary artery disease: the CLARICOR trial. Scand J Clin Lab Invest 2014; 74:657-664.

[18]. Nascimento MM, Hayashi SY, Riella MC, Lindholm B. Elevated levels of plasma osteoprotegerin are associated with all-cause mortality risk and atherosclerosis in patients with stages 3 to 5 chronic kidney disease. Braz J Med Biol Res 2014; 47 (11): 995-1002.
[19]. Evrard S, Delanaye P, Kamel S, Cristol JP, Cavalier E: Vascular calcification: from pathophysiology to biomarkers. Clin Chim Acta $2015 ; 438: 401-414$

[20]. Daniele G, Winnier D, Mari A, Bruder J, Fourcaudot M, Pengou Z, Hansis-Diarte A, Jenkinson C, Tripathy D, Folli F. The potential role of the osteopontin-osteocalcin-osteoprotegerin triad in the pathogenesis of prediabetes in humans. Acta Diabetol 2018; 2:139-148.

[21]. Lenora J, Ivaska KK, Obrant KJ, Gerdhem P. Prediction of bone loss using biochemical markers of bone turnover. Osteoporos Int 2007; 18: 1297-1305.

[22]. Zhang Y, Qi L, Gu W, Yan Q, Dai M, Shi J, Zhai Y, Chen Y, Liu J, Wang W, Ning G, Hong J: Relation of serum osteocalcin level to risk of coronary heart disease in Chinese adults. Am J Cardiol 2010; 106: 14611465.

[23]. Bao Y, Zhou M, Lu Z, Li H, Wang Y, Sun L, Gao M, Wei M, Jia W: Serum levels of osteocalcin are inversely associated with the metabolic syndrome and the severity of coronary artery disease in Chinese men. Clin Endocrinol 2011; 75:196-201.

[24]. Osorio A, Ortega E, Torres JM, Sanchez P, Ruiz-Requena E. Biochemical markers of vascular calcification in elderly hemodialysis patients. Mol Cell Biochem 2013; 374:21-27.

[25]. Lerchbaum E, Schwetz V, Pilz S, Grammer TB, Look M, Boehm BO, Obermayer Pietsch B, Marz W Association of bone turnover markers with mortality in men referred to coronary angiography. Osteoporos Int 2013; 24:1321-1332.

[26]. Lee HG, Choi HY, Bae JS. Endocan as a potential diagnostic or prognostic biomarker for chronic kidney disease. Kidney Int. 2014; 86 (6):1079-1081.

[27]. Bennett MR, Nehus E, Haffner C, Ma Q, Devarajan P. Pediatric reference ranges for acute kidney injury biomarkers. Pediatr Nephrol $2015 ; 4: 677-85$.

[28]. Uwaezuoke SN, Ayuk AC, Muoneke VU, Mbanefo NR. Chronic kidney disease in children: Using novel biomarkers as predictors of disease. Saudi J Kidney Dis Transpl 2018; 4: 775-784.

[29]. Cen C, Aziz M, Yang WL, Nicastro JM, Coppa GF, Wang P. Osteopontin Blockade Attenuates Renal Injury After Ischemia Reperfusion by Inhibiting NK Cell Infiltration. Shock. 2017; 1: 52-60.

[30]. Roos JF, Doust J, Tett SE, Tett SE, Carl MJ, Kirkpatrick CMJ. Diagnostic accuracy of cystatin C compared to serum creatinine for the estimation of renal dysfunction in adults and children a meta-analysis. Clin Biochem 2007; 40:383-91.

[31]. Ichihara K, Saito K, Itoh Y. Sources of variation and reference intervals for serum cystatin $\mathrm{C}$ in a healthy Japanese adult population. Clin Chem Lab Med. 2007; 45:1232-6.

[32]. Ferguson TW, Komenda P, Tangri N. Cystatin C as a biomarker for estimating glomerular filtration rate. Curr Opin Nephrol Hypertens 2015; (3): 295-300

[33]. Briguori C, Visconti G, Rivera NV, Focaccio A, Golia B, Giannone R, Castaldo D, De Micco F, Ricciardelli B, Colombo A. Cystatin C and contrast-induced acute kidney injury. Circulation 2010; 19:2117-22.

[34]. Askenazi DJ, Ambalavanan N, Goldstein SL. Acute Kidney injury in critically ill newborns: What do we need to learn? Pediatric Nephrology. 2009; 24 (2):265-74.

[35]. Mori K, Lee HT, Rapoport D, Drexler IR, Foster K, Yang J, SchmidtOtt KM, Chen X, Yi Li J, Weiss S, Mishra Y, Cheema FH, Markowitz G, Suganami T, Sawai K, Mukoyama M, Kunis C, D’Agaty V, Debaragn P, Barasch J. Endocytic delivery of lipocalin-siderophoreiron complex rescues the kidney from ischemia-reperfusion injury. $\mathrm{J}$ Clin Invest 2005; 115:610-21.

[36]. Mishra J, Dent C, Tarabishi R, Mitsnefes MM, Ma Q, Kelly C, Ruff SM, Zahedi K, Shao M, Bean J, Mori K, Barasch J, Devarjan P. Neutrophil gelatinase-associated lipocalin (NGAL) as a biomarker for acute renal injury after cardiac surgery. Lancet. 2005; 365:1231-8. 
[37]. Vera-Aviles M, Vantana E, Kardinasari E, Koh NL, Latunde-Dada GO. Protective Role of Histidine Supplementation against Oxidative Stress Damage in the Management of Anemia of Chronic Kidney Disease. Pharmaceuticals (Basel) 2018; 4: 2-15.

[38]. Castillo R, Huerta P, Carrasco R, Ramón R. Estrés oxidativo y daño renal. CIMEL Ciencia e Investigación Médica Estudiantil Latinoamericana 2003; 1:44-53.

[39]. Lee HS, Kim YS. Identification of oxidized low density lipoprotein in human renal biopsies. Kidney Int 1998; 3:848-56.

[40]. Sandhu S, Wiebe N, Fried LF, Tonelli M. Statins for improving renal outcomes: a meta-analysis. J Am Soc Nephrol 2006; 7:2006-16.

[41]. Gérard-Monnier D, Erdelmeier I, Régnard K, Moze-Henry N, Yadan JC, Chaudière J. Reactions of 1-methyl-2-phenylindole with malondialdehyde and 4 hydroxyalkenals. Analytical applications to a colorimetric assay of lipid peroxidation. Chem Res Toxicol 1998; $10: 1176-83$.

[42]. Oh JA, Shin HS. Simple and sensitive determination of malondialdehyde in human urine and saliva using UHPLC-MS/MS after derivatization with 3,4 diaminobenzophenone. J Sep Sci 2017; 20:39583968.

[43]. Gugliucci A, Kotani K, Kimura S. Paraoxonase 1 in chronic kidney failure. J Lipids 2012; 2012:1-10.

[44]. Noce A, Fabrini R, Dessì M, Bocedi A, Santini S, Rovella V, Pastore A, Tesauro M, Bernardini S, Di Daniele N, Ricci G. Erythrocyte glutathione transferase activity: a possible early biomarker for blood toxicity in uremic diabetic patients. Acta Diabetol 2014; 51(2): 219-24.

[45]. Lind C, Cadenas E, Hochstein P, Ernster L. DT-diaphorase: purification, properties, and function. Methods Enzymol 1990; 186: 287-301.

[46]. Anand U, Anand CV. Myeloperoxidase: a new twist to an old tale. India Clin Biochem 2012; 27(2):107-9.

[47]. Sokolov AV, Kostevich VA, Runova OL, Gorudko IV, Vasilyev VB, Cherenkevich SN, et al. Proatherogenic modification of LDL by surface-bound myeloperoxidase. Sokolov AV, Chem Phys Lipids 2014; 180:72-80. 